\title{
腾冲嗜酸两性菌硫氧化还原酶分子结构中铁原子 结合位点的新特征
}

陈志伟, 姜成英, 刘双江

中国科学院微生物研究所, 北京 100101

*联系人, E-mail: liusj@im.ac.cn

2008-10-01 收稿, 2008-12-11 接受

国家自然科学基金(批准号: 30670018, 30621005)资助项目

Chen Z W, Jiang C Y, Liu S J. Site-directed mutagenesis reveals new and essential elements for iron-coordination of the sulfur oxygenase reductase from the acidothermophilic Acidianus tengchongensis. Chinese Science Bulletin, 2009, 54(4): 652-657 doi: $10.1007 /$ s11434-009-0060-3

摘要 前期研究表明, 铁原子对于嗜酸两性菌中硫氧化还原酶(SOR)的活性至关重要. 本研 究表明, 2,2'-联吡啶、1,2-二羟基苯-3,5-二磺酸钠、8-差基喹啉等特异性铁离子螯合剂强烈抑 制腾冲嗜酸两性菌 SOR 酶活性, 进一步表明铁原子是 SOR 酶活必需. 对目前基因组数据库中 的 SOR 基因或者 SOR 类似基因进行序列比对, 发现了一个潜在的铁原子结合模体 $\left(\mathrm{H}^{86}-\mathrm{X}_{3}\right.$ $\left.\mathrm{H}^{90}-\mathrm{X}_{n}-\mathrm{E}^{114}-\mathrm{X}_{n}-\mathrm{E}^{129}\right)$. 据此, 本研究采用定点突变技术, 将氨基酸残基 $\mathrm{H}^{86}, \mathrm{H}^{90}$ 和 $\mathrm{E}^{129}$ 分别突变 为苯丙氨酸或者丙氨酸, 圆二色光谱测定发现突变体 $\left(\mathrm{H}^{86} \mathrm{~F}, \mathrm{H}^{90} \mathrm{~F}\right.$ 和 $\left.\mathrm{E}^{129} \mathrm{~A}\right)$ 的二级结构没有明 显改变, 但是这 3 个突变体全部丧失了酶活性. 突变体蛋白中铁原子含量测定结果表明, 3 个 突变体全部或者部分丢失了铁原子, 而之前研究中获得的 3 个半胱氨酸突变体(完全丧失了酶 活) 铁原子含量没有变化. 根据本研究并结合前期实验结果可知, SOR 分子中模体 $\mathrm{C}^{31}-\mathrm{X}_{n}-\mathrm{C}^{101}-\mathrm{X}_{2}-\mathrm{C}^{104}$ 是底物硫分子活化区域; 而模体 $\mathrm{H}^{86}-\mathrm{X}_{3}-\mathrm{H}^{90}-\mathrm{X}_{23}-\mathrm{E}^{114}-\mathrm{X}_{14}-(\mathrm{E} / \mathrm{D})^{129}$ 是 $\mathrm{SOR}$ 分 子中铁原子的结合区域, 与铁原子结合形成一个非卟啉铁中心, 是 SOR 的氧化还原中心; 这 两个区域均是 SOR 酶活性的必需部分.

关键词

嗜酸嗜热古菌 腾冲嗜酸两性菌 硫氧化还原酶 硫代谢 非卟啉铁中心
元素硫 $\left(\mathrm{S}^{0}\right)$ 的生物氧化还原作用是地球上硫元素 生物地质化学循环中的重要反应. 在富含硫元素的 陆地或深海热泉中经常可以检测并分离到与元素硫 代谢相关的嗜热酸古菌, 如硫化叶菌(Sulfolobus)和 嗜酸两性菌(Acidianus) ${ }^{[1 \sim 4]}$, 说明这些微生物在环境 中扮演着重要的角色. 硫化叶菌属和嗜酸两性菌属 的物种通过氧化元素硫获得能量, 这一过程的第一 步反应一一元素硫氧化是通过硫氧化还原酶(sulfur oxygenase reductase, SOR)完成的. 至今, 研究人员已 在Acidianus ambivalens ${ }^{[5,6]}$, Acidianus tengchongensis (同Acidianus sp. strain S5) ${ }^{[7 \sim 9]}$, Acidianus brieleyi ${ }^{[10]}$ 以及 Sulfolobus tokodai $\frac{111]}{1}$ 发现并证实了 SOR的存
在.

在分子氧存在的条件下, SOR催化元素硫歧化生 成亚硫酸、硫代硫酸和硫化氢 ${ }^{[5,10]}$. 这个酶是一个含 有 24 个相同亚基的多聚体, 单体分子量为 $35 \mathrm{kD}^{[5,9]}$, 不需辅酶或电子供体/受体参与反应 ${ }^{[12,13]}$. 鉴于元素 硫的特殊性质(疏水性和难溶性, $5 \mu \mathrm{g} / \mathrm{L}, 20^{\circ} \mathrm{C}$ ) ${ }^{[12]}$, 以 及此酶在嗜热酸古菌硫氧化代谢的关键性, 人们越 来越关注硫氧化还原酶的反应机理. 本实验室的前 期研究表明, SOR中的 3 个半胱氨酸残基 $\left(\mathrm{C}^{31}, \mathrm{C}^{101}\right.$ 和 $\mathrm{C}^{104}$, 编号参考腾冲嗜酸两性菌的SOR序列)为酶的 必需氨基酸残基 ${ }^{[14]}$.

通过对已知或推测的SOR 分子的氨基酸序列进 
行比对, 发现了一个潜在的铁离子结合位点- $-\mathrm{H}^{86}$ $\mathrm{X}_{3}-\mathrm{H}^{90}$ 和 $\mathrm{E}^{129}$ 模体. 近期的研究表明, $\mathrm{H}^{86}, \mathrm{H}^{90}$ 和 $\mathrm{E}^{114}$ 为Acidianus ambivalens硫氧化还原酶中铁离子的结 合位点 ${ }^{[15]}$. 在本研究中, 我们进一步证实了在腾冲嗜 酸两性菌的SOR中 129 位的谷氨酸残基 $\left(\mathrm{E}^{129}\right)$ 也为铁 离子的结合位点和酶活性的必需基团.

\section{1 材料与方法}

(i ) 菌株、质粒和培养条件. 本研究所使用的 菌株和质粒见表 1 , 大肠杆菌菌株在 $37^{\circ} \mathrm{C}$ 条件下用 $\mathrm{LB}$ 液体或固体培养基进行培养, 对于含有质粒的菌 株则添加 $100 \mu \mathrm{g} / \mathrm{mL}$ 的氨芐青霉素. 菌株的生长通过 可见分光光度计进行测定 $\left(A_{600 \mathrm{~nm}}\right)$. 硫氧化还原酶及 其突变基因克隆于 pBV220 载体并于 HB101 菌株中 表达. 重组菌株先在 $30^{\circ} \mathrm{C}$ 条件下培养至 $A_{600 \mathrm{~nm}}$ 约为 0.6 , 马上转入 $42^{\circ} \mathrm{C}$ 水浴摇床中继续震荡表达 $8 \mathrm{~h}$. 表 达的菌体离心收集 $(8000 \times g)$ 后保存于 $-73^{\circ} \mathrm{C}$.

(ii ) DNA 聚合酶、限制性内切酶和其他化合物. Pyrobest DNA 聚合酶(高保真 DNA 聚合酶)和 dNTPs 购于 TaKaRa，限制性内切酶购于 TaKaRa 或 NEB. 2,2'-联吡啶、1,2-二羟基苯-3,5-二磺酸钠、8-差基喹 㕲和 BCA 蛋白检测试剂盒购于 Sigma. 碱性磷酸酶 标记的羊抗兔二抗购于北京中山生物科技. （iii）蛋白提取和纯化. 蛋白粗提物的制备如文 献 $[9,13]$ 所述. 含有 SOR或其突变体的蛋白粗提物先 置于 $75^{\circ} \mathrm{C}$ 水浴中热处理 $15 \mathrm{~min}$, 然后高速离心 $(15000 \times g) 10 \mathrm{~min}$ 以去除大部分变性的大肠杆菌蛋白. 所得的上清液 (含有大部分的重组蛋白)导入 Hitrap ${ }^{\mathrm{TM}}$ Q HR $5 \mathrm{~mL}$ 的阴离子交换柱(用 $20 \mathrm{mmol} / \mathrm{L}$ Tris- $\mathrm{HCl}$ $\mathrm{pH} 7.5$ 预平衡)进行纯化, 重组蛋白通过氯化钠梯度 $(0 \sim 1 \mathrm{~mol} / \mathrm{L})$ 洗脱, 收集洗脱组分, 野生型测定酶活性, SOR突变体则通过SDS-PAGE和Western blotting检测 [9]. 所得的重组蛋白组分合并, 用超滤管(Vivaspin20，100 kD，Sartarius)浓缩，然后用Superdex ${ }^{\mathrm{TM}} 200$ $\mathrm{HR}$ (用 $20 \mathrm{mmol} / \mathrm{L}$ Tris- $\mathrm{HCl} \mathrm{pH} 7.5$ 预平衡)进一步纯化. 所用的蛋白纯化步骤均于 FPLC系统上运行 (Äkta FPLC, GE Healthcare). 纯化所得的蛋白超滤浓缩后 保存于 $-20^{\circ} \mathrm{C}$.

(iv) 定点突变和常规DNA操作. 用于定点突变 的引物序列均列于表 $1 . \mathrm{H}^{86}, \mathrm{H}^{90}$ 和 $\mathrm{E}^{129}$ 位点的突变通 过全质粒扩增和 Dpn I 篮选的方法进行 ${ }^{[16]}$. 突变的基 因通过活性测定并进一步测序鉴定. 常规的DNA操作, 如质粒提取、酶切和转化等, 参考文献[16].

（v）蛋白浓度和 SOR 酶活性测定. 蛋白浓度 通过 BCA 蛋白测定试剂盒测定(BCA1, Sigma). 硫氧

表 1 本研究中使用的菌株、质粒和引物

\begin{tabular}{|c|c|c|}
\hline 引物 & 性质或序列 & 来源或参考文献 \\
\hline \multicolumn{3}{|l|}{ 菌株 } \\
\hline HB101 & 硫氧化还原酶及其突变体的表达菌株 & \\
\hline XL1-blue & 用于一般转化和定点突变 & Stratagene 公司 \\
\hline \multicolumn{3}{|l|}{ 质粒 } \\
\hline pBV220sor & 野生型硫氧化还原酶表达质粒 & [7] \\
\hline pBV220sor/H86F & 硫氧化还原酶突变体 $(\mathrm{H} 86 \mathrm{~F})$ 表达质粒 & 本研究 \\
\hline pBV220sor/H90F & 硫氧化还原酶突变体(H90F)表达质粒 & 本研究 \\
\hline pBV220sor/E129A & 硫氧化还原酶突变体(E129A)表达质粒 & 本研究 \\
\hline pBV220sor/C31S & 硫氧化还原酶突变体(C31S)表达质粒 & 本研究 \\
\hline pBV220sor/C101S & 硫氧化还原酶突变体(C101S)表达质粒 & 本研究 \\
\hline pBV220sor/C104S & 硫氧化还原酶突变体 $(\mathrm{C} 104 \mathrm{~S})$ 表达质粒 & 本研究 \\
\hline \multicolumn{3}{|l|}{ 引物 ${ }^{\text {a) }}$} \\
\hline $\mathrm{H} 1 \mathrm{~F}$ & GTGGAAAGATTGGAAAGATTTTGAAGAAATGCACAGACAAAAC, 用于H86F突变 & 本研究 \\
\hline $\mathrm{H} 1 \mathrm{R}$ & GTTTTGTCTGTGCATTTCTTCAAAATCTTTCCAATCTTTCCAC, 用于H86F突变 & 本研究 \\
\hline $\mathrm{H} 2 \mathrm{~F}$ & GGAAAGATCATGAAGAAATGTTCAGACAAAACTGGAGTTACC, 用于H90F突变 & 本研究 \\
\hline $\mathrm{H} 2 \mathrm{R}$ & GGTAACTCCAGTTTTGTCTEAACATTTCTTCATGATCTTTCC, 用于H90F突变 & 本研究 \\
\hline E-F & GACATGCCAATAAACACTGCGATGACAGATTTCACTGC，用于E129A突变 & 本研究 \\
\hline E-R & GCAGTGAAATCTGTCATCGCAGTGTTTATTGGCATGTC，用于E129A突变 & 本研究 \\
\hline
\end{tabular}

a) 突变位点用下画线标示 
化还原酶的氧化酶活性测定如之前所述 ${ }^{[14]}$. 酶活单 位为 $1 \mathrm{~min}$ 产生 $1 \mu \mathrm{mol} / \mathrm{L}$ 氧化产物(亚硫酸和硫代硫 酸)所需的酶量. 亚硫酸和硫代硫酸的浓度分别通过 碱性品红和亚甲基蓝方法测定 ${ }^{[14]}$.

( vi ) SDS-PAGE和Western blotting. 重组蛋白的 SDS-PAGE电泳参照Laemmli ${ }^{[17]}$ 的方法, Western blotting参照文献[9].

（vii）铁离子及其他化合物对 SOR 酶活性的影响. $2,2^{\prime}$-联吡啶 $\left(2,2^{\prime}\right.$-dipyridyl) 是二价铁离子的特异螯合 剂, 而三价铁离子则为 1,2-二羟基苯-3,5-二磺酸钠 (4,5-dihydroxy- $m$-benzenedisulfonic acid, Tiron)和 8差基喹啉 (8-hydroxyquinoline，8-HQ)所特异螯合. 为了检测铁离子和这些铁离子特异螯合剂对 SOR 酶 活性的影响, 在酶反应体系中加入不同浓度的这些离 子和化合物(表 2), SOR 蛋白量为 $10 \mu \mathrm{g}(5 \mathrm{~mL}$ 反应体 系). 在室温下放置 $15 \mathrm{~min}$, 然后转入 $75^{\circ} \mathrm{C}$ 水浴中反应 $30 \mathrm{~min}$. 对照为不加化合物或不加酶的反应体系.

表 2 铁离子(二价和三价)及其特异螯合剂对硫氧化 还原酶活性的影响 a)

\begin{tabular}{lcc}
\hline \multicolumn{1}{c}{ 化合物 } & 浓度 $/ \mathrm{mmol} \cdot \mathrm{L}^{-1}$ & 残留活性 $(\%)$ \\
\hline 对照 & - & 100.0 \\
$\mathrm{Fe}^{2+}$ & 1.0 & 31.5 \\
$\mathrm{Fe}^{3+}$ & 1.0 & 0.3 \\
$2,2^{\prime}$-联吡啶 & 0.04 & 21.1 \\
& 0.2 & 0.0 \\
Tiron $^{\text {b) }}$ & 0.1 & 55.0 \\
& 1.0 & 52.8 \\
8-羟基喹啉 & 0.1 & 44.0 \\
& 1.0 & 17.8 \\
2,2'-联吡啶/超滤 & 10.0 & 199.4 \\
Tiron/超滤 & 10.0 & 239.7 \\
\hline
\end{tabular}

a) 2,2'-联吡啶 $(200 \mathrm{mmol} / \mathrm{L}) 、 1,2$-二羟基苯-3,5-二磺酸钠 (Tiron)(500 mmol/L)和 8 -羟基喹啉 $(500 \mathrm{mmol} / \mathrm{L}$ )分别溶解于二甲 基亚砜(DMSO)、 $50 \mathrm{mmol} / \mathrm{L}$ Tris- $\mathrm{HCl}$ 缓冲液( $\mathrm{pH}$ 7.5)和乙醇. 每一 个测定做 3 个平行. b) Tiron 对酶活性测定有影响

(viii) SOR 及其突变体铁含量测定. SOR 及其突 变体的纯化如前所述, 但需特别注意防止外源铁离 子的污染. 缓冲液使用超纯 Tris (Cat. No. 75825, USB)和色谱纯的盐酸(北京益利)配制, 所有的玻璃 容器均用色谱纯的硝酸 $(50 \%$, 用超纯水稀释)洗 3 遍. 重组蛋白超滤浓缩后以透过液作为空白对照.

蛋白和缓冲液的铁离子浓度通过原子发射光谱 (ICP-AES, IRIS Advantage, Thermo Jarrell Ash Co.)测 定(北京普尼物化分析中心). $3 \mathrm{mg}$ 的蛋白用色谱纯硝
酸消化, 得到 $10 \mathrm{~mL}$ 消化液. 取 $3 \mathrm{~mL}$ 消化液进行测 定, 每个样品设 3 个平行组. 仪器测定的参数: 蠕动 原流速为 $1.85 \mathrm{~mL} / \mathrm{min}$; 功率为 $1150 \mathrm{~W}$; CID 检测器 温度为 $-50.2^{\circ} \mathrm{C}$; 喷雾器压力为 $22 \mathrm{psi}(1 \mathrm{psi}=0.155$ $\mathrm{cm}^{-2}$ ); 铁离子的检测波长为 $259.94 \mathrm{~nm}$. 此条件下的 仪器检测极限为 $2 \mathrm{ppb}$ (parts per billion).

\section{2 实验结果}

\section{1 铁离子及其特异螯合剂对 SOR 酶活性的影响}

从表 2 可以看出, 二价和三价铁离子对硫氧化还 原酶的活性都有强烈的抑制作用，尤其是三价铁离 子, 在浓度为 $1 \mathrm{mmol} / \mathrm{L}$ 时就能使 SOR 几乎完全失活 (仅剩余 $0.3 \%$ 的酶活性). 二价铁离子的特异螯合剂 $2,2^{\prime}$-联吡啶能在低浓度下 $(0.2 \mathrm{mmol} / \mathrm{L})$ 使 SOR 完全失 活; 而对于三价铁离子的特异螯合剂，1,2-二羟基苯-

3,5-二磺酸钠和 8-差基喹啉, 在 0.1 或者 $1 \mathrm{mmol} / \mathrm{L}$ 的 浓度下能够使 SOR 活性下降 45\% 83.3\%. 当这些特 异螯合剂通过反复超滤和稀释清除以后, SOR 的活性 得到恢复, 甚至提高.

\subsection{SOR 及其突变体的构建和表达}

通过定点突变(全质粒扩增和 Dpn I 篮选)的方法 得到 SOR 的 3 个突变体 $\mathrm{H}^{86} \mathrm{~F}, \mathrm{H}^{90} \mathrm{~F}$ 和 $\mathrm{E}^{129} \mathrm{~A}$, 这些突 变基因转入大肠杆菌后, 热诱导重组大肠杆菌细胞 可以有效表达这些 SOR 或者 $\mathrm{SOR}$ 突变体 $\left(30 \sim 42^{\circ} \mathrm{C}\right)$. 经纯化后, 利用 SOR 蛋白特异性抗体, 经 Western blotting 证明表达的蛋白是 SOR 蛋白(图 1).

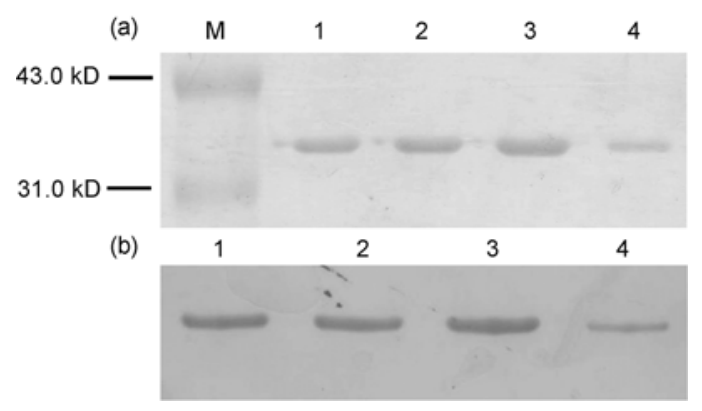

图 1 硫氧化还原酶及其突变体的 SDS-PAGE(a) 和 Western blotting 检测(b)

$\mathrm{M}$, 蛋白标准物; 1 , 硫氧化还原酶; $2, \mathrm{H}^{86} \mathrm{~F} ; 3, \mathrm{H}^{90} \mathrm{~F} ; 4, \mathrm{E}^{129} \mathrm{~A}$

\subsection{SOR 及其突变体的酶活性和铁含量}

如表 3 所示, SOR 分子的 $\mathrm{H}^{86}, \mathrm{H}^{90}$ 和 $\mathrm{E}^{129}$ 分别被 苯丙氨酸或丙氨酸取代后完全丧失了酶活性. 对比 
表 3 硫氧化还原酶及其突变体的活性和铁原子含量

\begin{tabular}{cccc}
\hline 酶 & $\begin{array}{c}\text { 铁原子含量/ } \\
\mathrm{mol} \cdot \mathrm{mol}^{-1} \text { 亚基 }\end{array}$ & $\begin{array}{c}\text { 铁原子残留率 } \\
(\% \pm \text { 相对标准偏差 })\end{array}$ & $\begin{array}{c}\text { 相对活性残留 } \\
\text { 率 }(\%)\end{array}$ \\
\hline $\mathrm{SOR}$ & 1.86 & $100 \pm 0.89$ & 100 \\
$\mathrm{C}^{31} \mathrm{~S}$ & 1.95 & $105 \pm 1.10$ & 0 \\
$\mathrm{C}^{101} \mathrm{~S}$ & 2.40 & $129 \pm 0.95$ & 1.6 \\
$\mathrm{C}^{104} \mathrm{~S}$ & 2.65 & $142 \pm 0.98$ & 0.8 \\
$\mathrm{H}^{86} \mathrm{~F}$ & 0 & $0 \pm 0.96$ & 0 \\
$\mathrm{H}^{90} \mathrm{~F}$ & 0.60 & $32.2 \pm 0.89$ & 0 \\
$\mathrm{E}^{129} \mathrm{~A}$ & 0.81 & $43.5 \pm 0.88$ & 0 \\
\hline
\end{tabular}

SOR 及其突变体的圆二色光谱(图 2), 表明其二级结 构没有发生明显变化, 说明这些突变体丧失酶活性 的原因不是由于蛋白分子结果变化引起的.

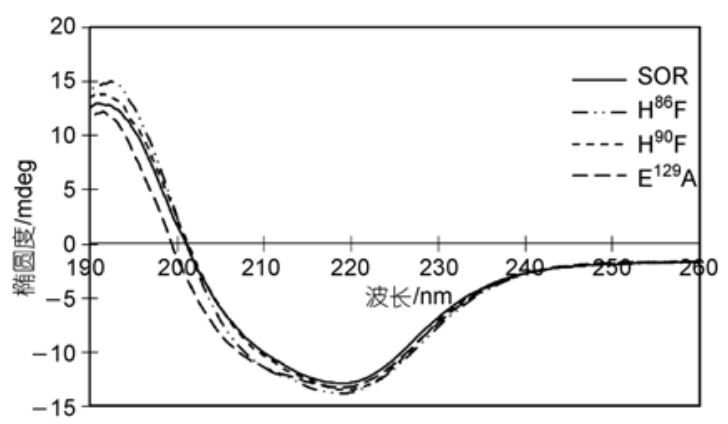

图 2 硫氧化还原酶及其突变体的圆二色光谱

这些突变体的二级结构没有明显变化. 蛋白浓度为 $100 \mu \mathrm{g} / \mathrm{mL}$, 扫描范围为 $190 \sim 260 \mathrm{~nm}$

SOR及其 6 个突变体 $\left(\mathrm{H}^{86} \mathrm{~F}, \mathrm{H}^{90} \mathrm{~F}, \mathrm{E}^{129} \mathrm{~A}, \mathrm{C}^{31} \mathrm{~S}\right.$, $\mathrm{C}^{101} \mathrm{~S}$ 和 $\mathrm{C}^{104} \mathrm{~S}$, 后 3 个突变体在之前的研究中获得 ${ }^{[14]}$ ) 的铁原子含量的测定结果表明(表 3), 半胱氨酸残基 突变体的铁原子含量与野生型基本一致, 但 $\mathrm{H}^{86} \mathrm{~F}$ 完 全不含铁原子, 而 $\mathrm{H}^{90} \mathrm{~F}$ 和 $\mathrm{E}^{129} \mathrm{~A}$ 的铁原子含量则有不 同程度的下降(分别仅为野生型的 $32.2 \%$ 和 $43.5 \%$ ).

\section{3 讨论}

以前的研究表明, SOR中可能含有一个低能量单 核的非卟啉铁中心, 而且这个非卟啉铁中心为 $\mathrm{SOR}$ 活性所必需; 同时, 在复性SOR的包涵体时必须加入 铁离子(二价或三价)才能得到有活性的酶分子 ${ }^{[12]}$. 本 研究结果表明铁离子(二价和三价)及其特异性螯合 剂对SOR酶活性有很强的抑制作用, 所以进一步对 其铁离子结合的必需氨基酸位点进行了研究. Urich 等人 ${ }^{[12]}$ 研究表明, 从A. ambivalens野生菌株中纯化和 在大肠杆菌重组表达的SOR中亚基的铁原子含量分 别为 1 和 2.2. 本研究结果表明, 重组的腾冲嗜酸两性 菌的SOR每摩尔亚基中含有 $1.86 \mathrm{~mol}$ 铁离子. 铁离子
(二价和三价)对SOR活性的结果表明, 过量的二价和 三价铁离子均对酶活性有明显抑制作用(表 2). 同时, 通过数次超滤和稀释去除过量的铁离子后SOR的活 性得到恢复, 表明铁离子的抑制作用并非铁离子对 SOR的修饰, 可能是过量的铁离子与SOR中的铁离子 中心发生竞争性抑制作用. 铁离子的特异性抑制剂 (2,2'-联吡啶、1,2-二羟基苯-3,5-二磺酸钠和 8-差基喹 啉)实验结果证实了酶分子中结合的铁原子为 $\mathrm{SOR}$ 活 性所必需, 但外加的过量铁离子则对酶活性有明显 抑制作用. 在反应体系中加入上述铁离子特异螯合 剂使SOR活性下降 45\% 100\%, 在随后实验中去除这 些铁离子特异螯合剂之后SOR的活性得到恢复甚至 提高.

非卟啉铁中心 $\left(H-\mathrm{X}_{3}-\mathrm{H}-\mathrm{X}_{\mathrm{n}}-\mathrm{E} / \mathrm{D}\right)$ 在邻苯二酚双加 氧酶、rieske铁硫蛋白双加氧酶、 $\alpha$-酮戊二酸依赖酶、 喋呤依赖水解酶以及其他一些氧化酶中广泛存在 ${ }^{[18]}$. 本研究和前期工作中对 SOR及其相似基因的氨基酸 序列进行比对和分析结果表明, SOR分子中存在一个 潜在的铁结合模体 $\mathrm{H}^{86}-\mathrm{E}-\mathrm{E}-\mathrm{M}-\mathrm{H}^{90}, \mathrm{E}^{114}$ 和 $\mathrm{E}^{129[13 \sim 15]}$. 对来源于 A. ambivalens硫氧化还原酶定点突变研究 表明, 这个非卟啉铁中心包括 $\mathrm{H}^{86}, \mathrm{H}^{90}$ 和 $\mathrm{E}^{114[13,15]}$, 然 而 $\mathrm{E}^{129}$ 的功能并没有得到证实. 在嗜酸两性菌和硫化 叶菌属的SOR基因中, 129 位的谷氨酸残基 $\left(\mathrm{E}^{129}\right)$ 是非 常保守的; 而在与嗜酸两性菌和硫化叶菌属亲缘较 远的Ferroplasma和Aquifex属中的SOR基因中, $\mathrm{E}^{129}$ 则 被与其性质相似的天冬氨酸残基所取代. 在本研究 中, $\mathrm{E}^{129}$ 和其他两个组氨酸残基 $\left(\mathrm{H}^{86}\right.$ 和 $\left.\mathrm{H}^{90}\right)$ 分别被取 代为丙氨酸或苯丙氨酸. 得到的 3 个突变体, $\mathrm{H}^{86} \mathrm{~F}$, $\mathrm{H}^{90} \mathrm{~F}$ 和 $\mathrm{E}^{129} \mathrm{~A}$, 都完全丧失了酶活性, 但它们对于铁 离子的结合作用却不尽相同: $\mathrm{H}^{86} \mathrm{~F}$ 完全不含铁离子, 而另外两个突变体一 $\mathrm{H}^{90} \mathrm{~F}$ 和 $\mathrm{E}^{129} \mathrm{~A}$ 则还残留 $32.2 \%$ 和 $43.5 \%$ 的铁离子含量. SOR以这些突变体的圆二色 光谱(图 2)比较表明, 这些位点的突变没有改变 SOR 的二级结构, 所以这些突变体失活的原因, 可能是由 于改变了 SOR分子中与催化相关的氨基酸残基. 假 如铁离子(二价或者三价)的结合位点是这些氨基酸 残基, 那么就很容易理解如果这些氨基酸残基被不 含电荷的其他氨基酸残基所取代的话, 相应的这些 突变体的铁离子含量就会下降甚至丧失. 本研究也 表明与 SOR活性密切相关的三个半胱氨酸残基 $\left(C^{31}\right.$, $\mathrm{C}^{101}$ 和 $\left.\mathrm{C}^{104}\right)$ 并没有参与铁离子的结合, 这是由于这 3 个突变体的铁离子含量与野生型相比并没有明显变 
化.

本研究及前期研究表明, 3 个半胱氨酸残基 $\left(\mathrm{C}^{31}\right.$, $\mathrm{C}^{101}$ 和 $\left.\mathrm{C}^{104}\right), \mathrm{H}^{86}, \mathrm{H}^{90}$ 和 $\mathrm{E}^{129}$ 为 $\mathrm{SOR}$ 活性所必需. 如同 Acidithiobacillus 和Acidiphilium中的硫氧化酶 ${ }^{[19]}$, 腾 冲嗜酸两性菌的SOR中 3 个半胱氨酸残基可能是底 物元素硫活化和结合的位点. 而保守模体 $\mathrm{H}^{86}-\mathrm{X}_{3}$ -
$\mathrm{H}^{90}-\mathrm{X}_{23}-\mathrm{E}^{114}-\mathrm{X}_{14}-\mathrm{E}^{129}$ 则可能作为单核亚铁的配基形 成非卟啉铁中心, 就像恶臭假单胞菌 (Pseudomonas putida)甲苯双加氧酶的非卟啉铁中心 $\left(\mathrm{E}^{214}, \mathrm{D}^{219}, \mathrm{H}^{222}\right.$ 和 $\left.\mathrm{H}^{228}\right)$ 一样, 活化分子氧, 催化底物氧化反应进行 [20]. 结合三维结构解析 ${ }^{[21]}$, SOR的催化机理还有待进 一步详细研究.

\section{参考文献}

1 Brock T D, Brock K M, Belly R T, et al. Sulfolobus: A new genus of sulfur-oxidizing bacteria living at low pH and high temperature. Arch Mikrobiol, 1972, 84(1): 54-68[doi]

2 Burton N P, Norris P R. Microbiology of acidic, geothermal springs of Montserrat: Environmental rDNA analysis. Extremophiles, 2000, 4(5): 315-320 [doi]

3 Segerer A, Neuner A M, Kristjansson J M, et al. Acidianus infernus gen. nov., sp. nov., and Acidianus brierleyi comb. nov.: Facultatively aerobic extremely acidophilic thermophilic sulfur-metabolizing archaebacteria. Int J Sys Bacteriol, 1986, 36(4): 559-564 Simmons S, Norris R. Acidophiles of saline water at thermal vents of Vulcano, Italy. Extremophiles, 2002, 6(3): 201 - 207 [doi]

5 Kletzin A. Coupled enzymatic production of sulfite, thiosulfate, and hydrogen sulfide from sulfur: Purification and properties of a sulfur oxygenase reductase from the facultatively anaerobic archaebacterium Desulfurolobus ambivalens. J Bacteriol, 1989, 171(3): $1638-1643$

6 Kletzin A. Molecular characterization of the sor gene, which encodes the sulfur oxygenase/reductase of the thermoacidophilic Archaeum Desulfurolobus ambivalens. J Bacteriol, 1992, 174(18): 5854-5859

7 He Z, Li Y, Zhou P, et al. Cloning and heterologous expression of a sulfur oxygenase/reductase gene from the thermoacidophilic archaeon Acidianus sp. S5 in Escherichia coli. FEMS Microbiol Lett, 2000, 193(2): 217-221 [doi]

8 He Z, Zhong H, Li Y. Acidianus tengchongensis sp. nov., a new species of acidothermophilic archaeon isolated from an acidothermal spring. Curr Microbiol, 2004, 48(2): 159-163 [doi]

9 Sun $\mathrm{CW}$, Chen Z W, He Z G, et al. Purification and properties of the sulfur oxygenase/reductase from the acidothermophilic archaeon, Acidianus strain S5. Extremophiles, 2003, 7(2): 131-134

10 Emmel T, Sand W, Koenig W A, et al. Evidence for the existence of a sulfur oxygenase in Sulfolobus brierleyi. J Gen Microbiol, 1986, 132: $3415-3420$

11 Liu S J. Archaeal and bacterial sulfur oxygenase reductase: Genetic diversity and physiological function. In: Dahl C, Friedrich C G, eds. Microbial Sulfur Metabolism. Heidelberg: Springer, 2008. 217-224

12 Urich T, Banerras T M, Leal S S, et al. The sulphur oxygenase reductase from Acidianus ambivalens is a multimeric protein containing a low-potential mononuclear non-haem iron center. Biochem J, 2004, 381(1): 137-146 [doi]

13 Urich T, Coelho R, Kletzin A, et al. The sulfur oxygenase reductase from Acidianus ambivalens is an icosatetramer as shown by crystallization and Patterson analysis. Biochim Biophys Acta, 2005, 1747(2): 267-270

14 Chen Z W, Jiang C Y, She Q X, et al. Key role of cysteine residues in catalysis and subcellular localization of sulfur oxygenase reductase of Acidianus tengchongensis. Appl Environ Microbiol, 2005, 71(2): 621-628 [doi]

15 Urich T, Kroke A, Bauer C, et al. Identification of core active site residues of the sulfur oxygenase reductase from Acidianus ambivalens by site-directed mutagenesis. FEMS Microbiol Lett, 2005, 248(2): 171-176 [doi]

16 Sambrook J, Russell D. Molecular Cloning: A Laboratory Manual. 3rd ed. Cold Spring Harbor: Cold Spring Harbor Laboratory Press, 2001

17 Laemmli U K. Cleavage of structural proteins during the assembly of the head of bacteriophage T4. Nature, 1970, 227(5259): 680-685 [doi]

18 Koehntop K D, Emerson J P, Que L J. The 2-His-1-carboxylate facial triad: A versatile platform for dioxygen activation by mononuclear non-heme iron (II) enzymes. J Biol Inorg Chem, 2005, 10(2): 87-93 [doi]

19 Rohwerder T, Sand W. The sulfane sulfur of persulfides is the actual substrate of the sulfur-oxidizing enzymes from Acidithiobacillus and Acidiphilium spp. Microbiology, 2003, 149(7): 1699-1709 [doi]

20 Jiang H, Parales R E, Lynch N A, et al. Site-directed mutagensis of conserved amino acids in the alpha subunit of toluene dioxygenase: Potential mononuclear non-heme iron coordination sites. J Bacteriol, 1996, 178(11): 3133-3139

21 Li M, Chen Z W, Zhang P, et al. Crystal structure studies on sulfur oxygenase reductase from Acidianus tengchongensis. Biochem Biophys Res Commun, 2007, 396(3): 919-923 